\title{
Call for Papers: Autism in Adulthood
}

\author{
Editor: Christina Nicolaidis, MD, MPH; Portland State University; Oregon Health \& Science University
}

A utism in Adulthood is the home for research and scholarship on the most pressing issues affecting adults on the autism spectrum, from emerging adulthood to later life. Autism in Adulthood welcomes original research, indepth analysis, and inter-professional dialogue from multiple disciplines, professions, methodologies, and paradigms.

\section{Coverage includes:}

- Physical and mental health of adults on the autism spectrum

- Employment, housing, education, community participation, economic well-being, and quality of life

- Sexuality, gender identity, relationships, and caregiving

- Autistic intelligence, sensory processing, communication, executive functioning, and flexibility

- Healthcare and social services

- Identification, treatment, and accommodation of co-occurring conditions

- Basic science research with implications for autism in adulthood

- Biological, neurological, psychological, cognitive, emotional, behavioral, and social changes with aging

- Policies and programs affecting adults on the autism spectrum

- Human and disability rights and advocacy

- Portrayal of adult autism in society and the media

- Ethical issues in research, practice, or policy on autism in adulthood

- Intersectionality of autism and race, ethnicity, nationality, gender, sexual orientation, disability, class, trauma, discrimination, and other social determinants of health

- Transitions across the lifespan, including transitions to and through adulthood

- Insights about autism from adults on the spectrum

- Participatory approaches to research, practice, and policy, in partnership with individuals on the spectrum

- Methodological advances for conducting research on autism in adulthood

Please read our Instructions for Authors at http://www.liebertpub.com/aut for complete details about coverage.

\section{Benefits of publishing in Autism in Adulthood include:}

- Fast and user-friendly electronic submission

- Rapid, high-quality peer review

- Maximum exposure: accessible in 170 countries

- Open access options available

- End-to-end Author Benefits Program
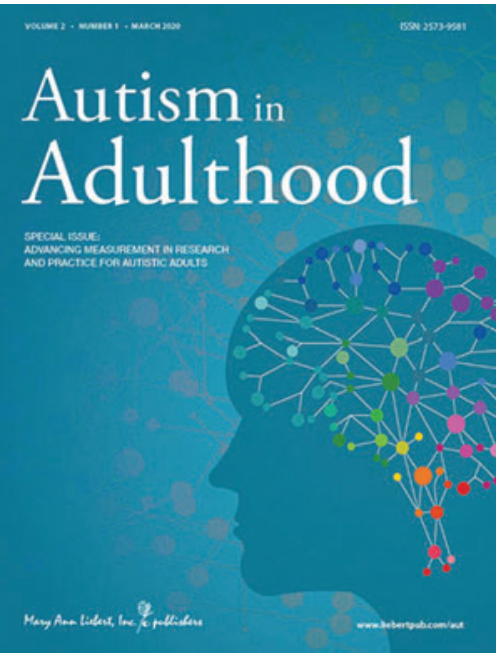

\section{Please visit the Instructions for Authors: www.liebertpub.com/aut}

\section{Submit your paper for peer review online: https://mc.manuscriptcentral.com/aut}

